\title{
Onychomycosis in Patients Living with HIV/AIDS
}

\author{
Gabriela Moreno-Coutiño ${ }^{1}$
}

Published online: 28 August 2019

(C) Springer Science+Business Media, LLC, part of Springer Nature 2019

\begin{abstract}
Purpose of Review As both onychomycosis and HIV/AIDS are frequently encountered, we aimed to make a review of the existent information to this moment, including classification, evaluation and treatment.

Recent Findings The new description of dermatophytes will surely originate changes in the classification and probably in the therapeutic approach. Also, we must be watchful for the modifications that may develop in this pathology with the newer antirretrovirals which are in constant evolution and could modify the patients' response to dermatophytosis.

Summary At this moment, we know that onychomycosis is very common in patients living with HIV.

Although they present similar clinical patterns and aetiological agents to the general population, we must focus in the many differences they present to further understand the physiopathology in the HIV setting.

We are still searching for the ideal antimycotic and the full understanding of the pathogenesis of the infection in this particular group of patients.
\end{abstract}

Keywords Onychomycosis $\cdot$ HIV/AIDS $\cdot$ Immunosuppression $\cdot$ Dermatophytes $\cdot$ Nail disease

\section{Introduction}

Since the introduction of cART (combined antiretroviral therapy), HIV-positive patients differ significantly from those already diagnosed with AIDS. These drugs have enabled HIVinfected individuals to have undetectable viral load and high CD4 cell counts, so their immunity is close to normal. Nonetheless, HIV infection continues to be a major public health problem with almost 37 million people suffering the disease, close to 2 million new infections/year, and only $70 \%$ of the affected ones are aware of their status. So, there are still many things to be done in order to control this pandemic [1].

On the other hand, onychomycosis is a chronic fungal infection of the nail plate, generally caused by dermatophytes that until recently, three genera were recognized

This article if part of the Topical Collection on Fungal Infections of Skin and Subcutaneous Tissue

Gabriela Moreno-Coutiño

gmorenocoutino@gmail.com

1 Mycology Section, General Hospital Dr. Manuel Gea González, Calzada de Tlalpan 4800 Colonia Sección XVI, 14080 Tlalpan Ciudad de México, CP, Mexico
(Trichophyton, Epidermophyton and Microsporum), but now, through phylogenetic studies, other genera have emerged [2] (Table 1). Despite these novelties, the clinical picture, diagnosis and treatment remain the same (Figs. 1 and 2).

It is the most common onychopathy, up to $50 \%$ of all nail diseases, and as it is mainly asymptomatic, it surely goes underdiagnosed. But, those patients that seek medical attention for this condition report reduced quality of life as this affects their interpersonal relationships and diminishes the self-esteem and they act as reservoirs for the fungus posing a risk to those in close contact with them. So, this infection is related to labour, economic and social problems.

Those affected have a wide spectrum of clinical manifestations. In 1972, Zaias et al. published a classification for onychomycosis that included four clinical forms. Recently, this was modified by adding one more form, so currently five are recognized: distal and lateral subungual onychomycosis (DLSO), superficial white onychomycosis (SWO), endonyx onychomycosis (EO), proximal subungueal onychomycosis (PSO) and total dystrophic onychomycosis (TDO); although in clinical practice, EO (the one recently added) is excluded for its complicated identification [3]. 
Table 1 New

classification of

dermatophytes

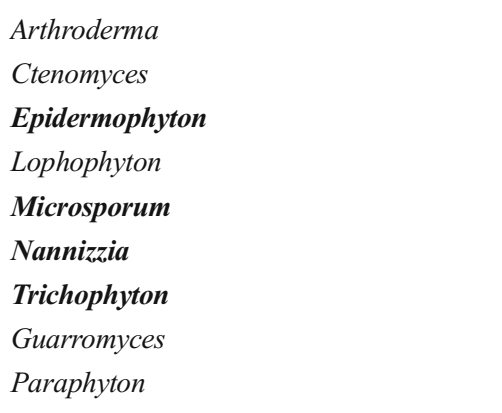

In bold are the genera that can affect humans

The most accepted theory states that the DLSO and TDO infections are acquired through the hyponychium. The less frequent forms have other theoretic incursion mechanisms. In SWO, the infection is initiated by the fungal invasion of the superficial nail plate. The most controverted form is PSO where two possible routes of infection have been proposed. The oldest one states that the fungi invade the nail plate through the proximal nail fold and cuticle. However, some authors think this is unlikely and have proposed the newer theory that involves a hematogenous/lymphatic route. This is based on the mechanisms present in the deep dermatomycosis, which are rare, but some lymph nodes of these patients have been studied and fungal structures identified in them. To further support this theory, they have noted that this clinical pattern can alternate infected and healthy transversal nail bands. This theory has not been proven, as the studies required for its comprobation would be too invasive for the patient [4••]. So, at the end, Baran et al. concluded that more than one route of infection may be possible for PSO.

Two scoring systems have been described, as an aid to classify the severity of the nail infection and they are mostly used for research purposes $[5,6]$.

When HIV/AIDS was initially recognized, a large list of comorbidities emerged. Onychomycosis was one of them, and Dompmartin et al. published one of the most read works in the

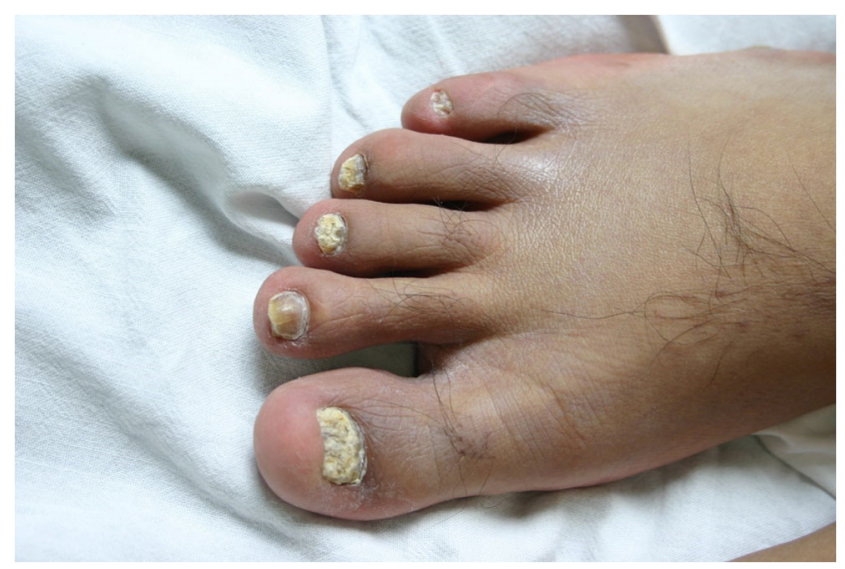

Fig. 1 TDO in all toenails

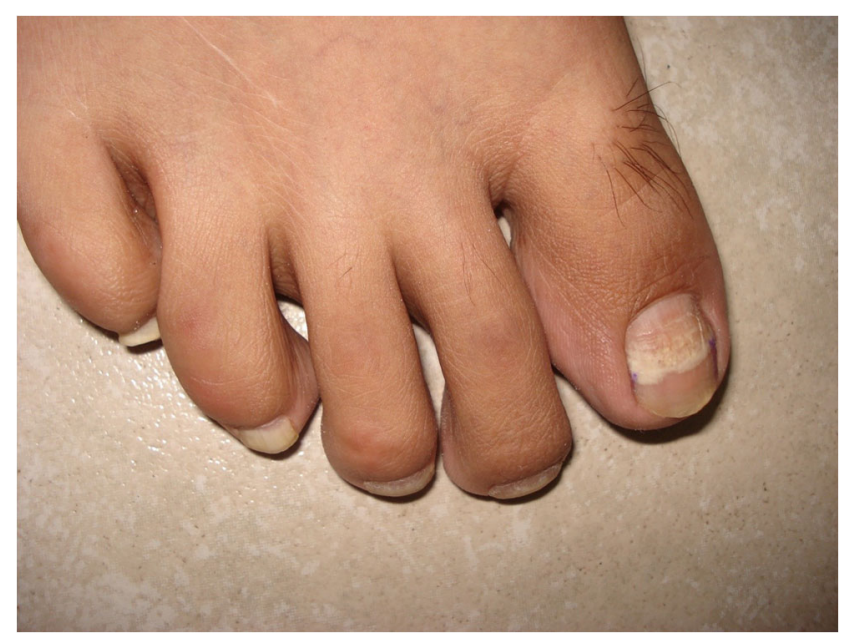

Fig. 2 SWO in toenails

1990s on this topic, where they describe very well the predominance in several nails, mostly toenails, and the rapid progression. They also reported that these patients had PSO as the predominant clinical form, and this statement misled for a long time with the idea that white onychomycosis are the prevailing clinical presentation [7].

Currently, the most common clinical form is DLSO, the same as in open population, although, if we encounter white onychomycosis or several affected nails with different clinical patterns, we should discard immunosuppression $[8 \cdot \bullet, 9]$.

Initially, a correlation between CD4 cell count and OM was reported and was used to classify patients, although now we know this interrelation is not so direct and nail infections can be seen within a wide range of CD4 cell count. The differences can be found regarding the severity of nail involvement as well as the number of affected nails [10].

The prevalence of OM varies depending on the studied population, with a reported range from 3.33 to $44 \%[11 \cdot 12]$.

In Mexico City, we found a higher incidence compared to India or China (21\% vs 3.33-11.9\% and 9.2\%, respectively) and they also had less than $450 \mathrm{CD} 4$ count, but did not find more involvement than those patients with less than $200 \mathrm{CD} 4$ $\mathrm{mm} 3[12,13,14 \cdot]$.

Some texts, particularly the first reports, describe a bad response to treatment and those relapses were more common than in the non-HIV population [10].

What we see now is that the antifungal treatment has the same effectiveness as in any other patient, and furthermore, these patients can improve or even cure the onychomycosis without antifungal treatment, by just receiving combined antiretroviral therapy (cART). These findings seem to support that the cellular immunity plays an important role in the eradication of the nail infection, although the precise mechanism still needs to be studied to further understand the involved factors that play a role or participate more actively in the cure or clinical improvement $[11 \bullet, 13]$. 
One of the already identified participant factor in the improvement/cure of onychomycosis without specific treatment is the fact that HIV-infected individuals have a higher rate of nail growth pace when compared to healthy controls matched by sex and age. This, at least partially can explain that the fungus has less time to invade the nail plate, that is one of the "protective" factors children have, as their nails grow faster than adults, and is the contrary to what is seen in the elderly, where the extremely slow nail growth rate, particularly in the toe nails, facilitate the infection [15].

Regarding the aetiology, the most frequently isolated agents are dermatophytes, particularly Trichophyton rubrum, which predominates in all clinical variants, although several case reports of onychomycosis caused by T. mentagrophytes and the NDM Talaromyces marnefei can be found [16].

Nonetheless, non-dermatophyte moulds (NDM) and Candida yeasts can also cause nail infection. Some reports mention Candida sp. as the aetiological agent found in finger and toenails $[12,17]$.

But most reports agree that the dermatophytes predominate, and the yeast-related onychomycosis are mostly anecdotic [13].

This will very likely change in the near future, as the dermatophytes are being reclassified and the number of species has increased.

\section{Diagnosis}

Traditionally, the diagnosis for onychomycosis is divided in two stages. The first one allows to observe the hyphae and permits to initiate the antifungal therapy. This is a direct exam, also called $\mathrm{KOH}$ mount. The second stage is the culture in Sabouraud agar with antibiotics that enables us to isolate and identify the aetiological agent.

So far, culture is still considered the gold standard, even though, in the best conditions, only $50 \%$ of the cultures will develop, and when they do, they can take up to 4 weeks to grow. Despite these downsides, this permits the specie identification with almost $100 \%$ specificity. The sensitivity is lower, with $56 \%$ average, within a wide range that varies between 29 and $82 \%$.

The main downside of the $\mathrm{KOH}$ mount is operator dependant and requires experience to identify the fungal structures [18•].

Another option for diagnosis that is particularly useful for those that do not have access to a mycology laboratory is the PAS stain of the hyponychium scale or distal nail trim processed as any other biopsy. This is highly sensitive (81-91\%) and specific. Some authors have even proposed this option as the gold standard arguing that this test has higher sensibility and specificity than the more traditional $\mathrm{KOH}$ mount and culture $[19,20]$.
However, we must remember that PAS staining does not allow the species identification and it is more expensive and time consuming compared to the $\mathrm{KOH}$ mount, so it is best considered as a second line tool for diagnosis [21, 22].

Following this line of thought, although less popular, we can apply to the same scale the Gomori-Grocott stain to the same sample with similar results, advantages and disadvantages [23].

So, in conclusion, a good option for diagnostic approach is to perform culture- and biopsy-stained scale/nail clipping.

Dermoscopy has been recently explored as a diagnosis tool for many dermatological diseases, including onychomycosis and other onychopathies. The longitudinal striae and the distal frayed border are seen in all the cases, with a spiked pattern predomination $(78 \%)$ and colour changes $(38 \%)$. This method's main disadvantage is being operator dependant, so currently the recommendation is to use it as a complement of any other diagnostic tool or to use it as a guide to obtain a better scale sample $[24,25]$.

Molecular essays are gaining relevance, mainly in the investigation and epidemiological areas. These tests are able to precisely identify the aetiological agents. They have good sensibility; however, the specificity is still low [22].

There are several useful techniques that act by amplifying the fungal DNA, such as nested or semi nested polymerase chain reaction (PCR), restriction fragment length polymorphism analysis (RFLP), loop-mediated isothermal amplification (LAMP), real-time PCR (qPCR), and also matrix-assisted laser desorption/ionization (MALDI-TOF) that through mass spectrometry are able to identify fungi [23].

It is always recommended to perform at least one, but preferably two diagnostic tests, as the clinical picture and the physical exam are frequently insufficient to support a diagnosis that requires the administration of systemic antifungal drugs for a lengthy period that has economic impact and possible drug interactions or adverse reactions.

\section{Differential Diagnosis}

Half of the onychopathies are not fungal infections, so it is extremely important to familiarize with the most frequent differential diagnosis.

As in the general population, traumatic onychodystrophy is one of the most common causes, particularly in the first and fifth toenails. Lichen planus and psoriasis are also to be considered, keeping in mind that these nails may be suprainfected by fungi and thus pose a diagnostic challenge.

Patients living with HIV/AIDS commonly suffer of human papilloma virus infection (HPV) in any of its clinical forms of presentation, and this includes ungual and periungual warts that may deform the nail plate. A biopsy will be required to confirm the diagnosis and also to discard the possibility of 
malignant transformation to epidermal carcinoma, another differential diagnosis for these cases.

Subungueal melanoma accounts for $2 \%$ of all melanomas in Caucasians. However, in black people and mestizos, acral melanoma (which includes palms, plants and subungueal areas) is the most common presentation, adding up to $70 \%$ of the melanomas in these groups. In Mexico, acral melanoma is reported to have an incidence between 24 and $31 \%$. In the initial stages, where colour changes predominate the clinical picture, and then the onychodistrophy manifests, is when it may be confused with onychomycosis [26]. Other diagnoses to keep in mind are pyogenic granuloma and epidermoid carcinoma [27].

For any onychodystrophy, as a general rule, onychomycosis is the first diagnosis to be discarded, as the diagnostic methods are non-invasive, fast and economic. In contrast, to confirm any differential diagnosis of onychomycosis, a nail biopsy is needed; this is an invasive and expensive approach, with the risk of leaving permanent dystrophy.

\section{Treatment}

The importance of treating onychomycosis is gaining importance every day, putting distance from the former idea of only cosmetic relevance, particularly for patients with chronic diseases and immunosuppression as the infected nails act as fungal reservoirs and tend to chronicity.

Throughout the literature, countless options for onychomycosis treatment are apparently available. However, only few are truly effective and suitable choices.

So, to decide the best treatment for a patient, we take into account several facts. We must know if the patient is taking antiretroviral drugs, and if so, since when. In my experience, it is favoured to wait for the first 6 months prior to initiating antifungal drugs for onychomycosis, for two main reasons. First, we know that antirretrovirals can cause several adverse effects, and these are better valuated with the least number of drugs simultaneously administered. The second reason is that we have seen that patients may improve or even cure the onychomycosis without antimycotics, and although the chances are low, it is worth the wait $[11 \bullet]$.

When the 6 months are over, and the patient is ready to receive antifungal drugs, we must assess the clinical presentation of the onychomycosis that might have varied from the first evaluation. As in the general population, superficial forms may be treated with topical medication only, where that lacquer's penetration to the nail plate is sufficient. But these cases are the least common. Most frequently, we find DLSO, which requires systemic administration, as well as in the PSWO and TDO forms.
The association of tinea with any clinical form of OM is common, particularly tinea pedis. These patients must receive appropriate treatment, as the fungal infection alters the cutaneous barrier and increases the risk of bacterial suprainfection or cellulitis.

The lacquers available are numerous, with active principles as ciclopiroxolamine and amorolfine that show similar cure rates. Many new drugs are being developed and commercialized but with comparable results as the older lacquers. So, research is being done in this area, since it is considered the best administration route, but still failure prevails in the penetration of the nail plate. These drugs may also be used as prophylaxis or coadyuvant in oral therapy.

Currently, the first choice for oral therapy is terbinafine, as it has shown to have the best clinical and mycological cure rates and, reportedly, also the least number of drug to drug interactions. The dosage and duration of the treatment is the same as in the open population, $250 \mathrm{mg}$ a $\mathrm{qd}$ for 3 months or pulses of 1 week of $500 \mathrm{mg}$ qd per month for 3 months.

The second line of oral treatment in the literature is my first line of treatment. Itraconazole has a wide spectrum, is more price accessible and does not interact with the main antiretroviral drugs employed nowadays. And also, if the patient has another mycotic systemic infection, or requires prophylaxis, he is likely to receive itraconazole, so sometimes only dosage adjustment has to be made.

Itraconazole is indicated at $200 \mathrm{mg}$ qd for 3 months or $400 \mathrm{mg} \mathrm{dq}$ for 1 week a month for 4 months, or even 6 months, which according to a report, increases the cure rates [28].

Fluconazole is not a feasible option; although reported as being useful, the treatment is extremely long, and thus, impractical.

Griseofulvin is still mentioned in articles as another therapeutical option, but actually it is not. It has a very narrow action spectrum, patients show many adverse reactions, and sometimes it is difficult to find [29•].

Although many authors have stated that these patients show a lower response rate to antifungal therapy, we do not share this appreciation $[12,30]$.

Among the few novelties of onychomycosis, therapy is an interesting proposal of antimycotic patches that are currently under evaluation, so we must wait for the results $[31 \cdot]$.

Due to the few available effective drugs, their cost and drug interactions, many people have tried alternate therapies that range from herbal to Vicks VapoRub ${ }^{\mathrm{TM}}$ and technologies such as laser. One study used the natural adhesive resin propolis, produced by bees (Apis mellifera), and has shown in vitro antibacterial and antifungal properties, with some capacity to penetrate the nail plate and to destroy the biofilm formed by Trichophyton spp. The 
clinical and mycological cure rate was $56 \%$, with a follow-up of 6 months [32]. Numerous articles have been published regarding laser therapies for onychomycosis, using many different frequencies. Some find certain clinical improvement, without mycological cure, so with these results and its high cost, so far, none has proven to be useful enough to recommend it [33]. A journal published an article of onychomycosis in patients living with HIV/AIDS treated with Vicks VapoRub ${ }^{\mathrm{TM}}$. They reported improvement in the majority of the participants and complete cure in two of them. However, they do not explain the way it was applied, the dosage or the mechanism of action. Also, they mention that the $\mathrm{KOH}$ mounts and cultures are negative in the follow-up visits, but the pictures in the article only show slight improvement, not to be qualified as cure. So, this option cannot be recommended either [30]. A recently published work used a chemical peel for the nail plate in patients that could not receive systemic antifungals with promising results. Hopefully, we will have more information soon [34•].

\section{Conclusions}

Onychomycosis is one of the most common dermatophytic infection and continues to rise as well as the HIV epidemic, so these diseases will frequently coexist.

Patients living with HIV/AIDS have particular characteristics regarding presentation and evolution of the onychomycosis that grants them to be analysed separately from other patients. Among these differences with general population, the most important one are that patients are younger, the number of involved nails is greater, fingernails more frequently comprised, not necessarily the toe's nail is affected, many clinical patterns may present simultaneously, the infection can rapidly advance in weeks, they have faster growing rate, and also they may cure or improve without specific treatment if they initiate cARV.

On the other hand, the similarities with the open population are the same aetiological agents, the clinical presentations, and the impact on the quality of life, as well as the diagnosis techniques and treatment options.

We still need a more useful clinical classification and better treatment options, so there is plenty of room for new research.

\section{Compliance with Ethical Standards}

Conflict of Interest Gabriela Moreno-Coutino declares no conflicts of interest relevant to this manuscript.

Human and Animal Rights and Informed Consent This article does not contain any studies with human or animal subjects performed by any of the authors.

\section{References}

Papers of particular interest, published recently, have been highlighted as:

- Of importance

- Of major importance

1. https://www.who.int/es/news-room/fact-sheets/detail/hiv-aids. 1004-2019.

2. De Hoog GS, Dukik K, Monod M, Packeu A, Stubbe D, Hendrickx $\mathrm{M}$, et al. Toward a novel multilocus phylogenetic taxonomy for the dermatophytes. Mycopathol. 2017;182:5-31. https://doi.org/10. 1007/s11046-016-0073-9.

3. Hay R, Baran R. Onychomycosis: a proposed revision of the clinical classification. J Am Acad Dermatol. 2011;65:1219-27. https:// doi.org/10.1016/j.jaad.2010.09.730.

4. - Baran R, Faergemann J, Hay RJ. Superficial White onychomycosis-a syndrome with different fungal causes and paths of infection. J Am Acad Dermatol. 2007;57:879-82. https://doi. org/10.1016/j.jaad.2007.05.026. This article proposes new infection routes for onychomycosis.

5. Sergeev AY, Gupta AK, Sergeev YV. The scoring clinical index for onychomycosis (SCIO index). Skin Therapy Lett. 2002;1:6-7.

6. Carney C, Tosti A, Daniel R, Scher R, Rich P, DeCoster J, et al. A new classification system for grading the severity of onychomycosis. Arch Dermatol. 2011;147:1277-82. https://doi. org/10.1001/archdermatol.2011.267.

7. Dompmartin D, Dompmartin A, Deluol AM, Grosshans E, Coulaud JP. Onychomycosis and AIDS. Int J Dermatol. 1990;29: $337-9$.

8.•• Moreno-Coutiño G, Arenas R, Reyes-Terán G. Improvement of onychomycosis after initiation of combined antiretroviral therapy. Int J Dermatol. 2013;52:311-3. https://doi.org/10.1111/j.13654632.2011.05346.x. New observations.

9. Gómez-Moyano E, Crespo-Erchiga V. HIV infection manifesting as proximal White onychomycosis. N Engl J Med. 2017;377(18):e26. https://doi.org/10.1056/NEJMicm1703082.

10. Daniel CR III, Norton LA, Scher RK. The spectrum of nail disease in patients with human immunodeficiency virus. J Am Acad Dermatol. 1992;27:93-7.

11. Ruíz-López P, Moreno-Coutiño G, Fernandez-Martínez R, Espinoza-Hernández J, Rodríguez Zulueta P, Reyes-Terán G. Evaluation of improvement of onychomycosis in HIV infected patients after initiation of combined antiretroviral therapy without antifungal treatment. Mycoses. 2015;58:516-21. https://doi.org/ $10.1111 /$ myc. 12350 . This article highlights the different characteristics of onychomycosis in HIV population and their particular evolution.

12. Jiménez-González C, Mata-Marin JA, Arroyo-Anduiza CI, Ascencio-Montiel IJ, Fuentes-Allen JL, Gaytán-Martínez J. Prevalence and aetiology of onychomycosis in the HIV infected population. Eur J Dermatol. 2013;23:378-81. https://doi.org/10. 1684/ejd.2013.2015.

13. Moreno-Coutiño G, Reyes-Terán G. Dermatosis en pacientes con VIH/SIDA en el Centro de Investigación de Enfermedades Infecciosas. Salud Pública Mex. 2015;57:486-7.

14. Vasudevan B, Sagar A, Bahal A, Mohanty AP. Cutaneous manifestations of HIV- a detailed study of morphological variants, markers of advanced disease, and the changing spectrum. Med J Armed Forces India. 2012;68:20-7. https://doi.org/10.1016/S03771237(11)60122-6. Good clinical review.

15. Sánchez-Moreno EC, Marioni-Manriquez S, Fernández Martínez $\mathrm{RF}$, Moreno-Coutiño G. Accelerated nail growth rate in HIV 
patients. Int J Dermatol. 2017;56:524-6. https://doi.org/10.1111/ijd. 13498.

16. Sunny N, Nair SP, Justus L, Beena A. Total dystrophic onychomycosis caused by Talaromyces marneffei in a patient with acquired immunodeficiency syndrome on combined anti-retroviral therapy. Indian J Dermatol Venereol Leprol. 2018;84:87-90. https://doi.org/10.4103/ijdvl.IJDVL_971_16.

17. Kaplan MH, Sadick N, McNutt S, Mètzer M, Sarngadharan, Pahwa S. Dermatologic findings and manifestations of acquired immunodeficiency síndrome (AIDS). J Am Acad Dermatol. 1987;16:485-506.

18. Velasquez-Agudelo V, Cardona-Arias JA. Meta-analysis of the utility of culture, biopsy, and direct $\mathrm{KOH}$ examination for the diagnosis of onychomycosis. BMC Infect Dis. 2017;17:166. https://doi.org/ 10.1186/s12879-017-2258-3. Good review.

19. Jeelani S, Ahmed QM, Lanker AM, Hassan I, Jeelani N, Fazili T. Histopathological examination of nail clippings using PAS staining (HPE-PAS): gold standard in diagnosis of onychomycosis. Mycoses. 2015;58:27-32. https://doi.org/10.1111/myc. 12251.

20. Hajar T, Fernández-Martínez R, Moreno-Coutiño G, Vásquez del Mercado E, Arenas R. Modified PAS stain: a new diagnostic method for onychomycosis. Rev Iberoam Micol. 2016;33:34-7. https:// doi.org/10.1016/j.riam.2014.10.002.

21. Mayer E, Izhak OB, Bergman R. Histopathological periodic acidschiff stains of nail clippings as a second-line diagnostic tool in onychomycosis. Am J Dermatopathol. 2012;34:270-3. https://doi. org/10.1097/DAD.0b013e318234cc49.

22. Nagar R, Nayak CS, Deshpande S, Gadkari RP, Shastri J. Subungual hyperkeratosis nail biopsy: a better diagnostic tool for onychomycosis. India J Dermatol Venereol Leprol. 2012;78:620 4. https://doi.org/10.4103/0378-6323.100579.

23. Ghannoum M, Mukherjee P, Isham N, Markinson B, Del Rosso J, Luis L. Examining the importance of laboratory and diagnostic testing when treating and diagnosing onychomycosis. Int $\mathrm{J}$ Dermatol. 2018;57:131-8. https://doi.org/10.1111/ijd.13690.

24. Nargis T, Pinto M, Shenoy MM, Hedge S. Dermoscopic features of distal lateral subungual onychomycosis. Indian Dermatol Online J. 2018;9:16-9. https://doi.org/10.4103/idoj.IDOJ_40_17.

25. Bet DL, dos Reis AL, Di Chiacchio N, Belda $\bar{W} \overline{J r}$. Dermoscopy and onychomycosis: guided nail abrasión for mycological samples. An Bras Dermatol. 2015;90:904-6. https://doi.org/10.1590/ abd1806-4841.20154615.
26. De la Fuente GA, Ocampo-Candiani J. Melanoma cutáneo. Gac Med Mex. 2010;146:126-35.

27. Riahi RR, Cohen PR, Goldberg LH. Subungual amelanotic melanoma masquerading as onychomycosis. Cureus. 2018;10(3):e2307. https://doi.org/10.7759/cureus.2307.

28. Zhang L, Xu H, Shj Y, Tao Y, Li X. An exploration of the optimum dosage and number of cycles of itraconazole pulse therapy for severe onychomycosis. Mycoses. 2018. https://doi.org/10.1111/myc. 12799.

29. Kreijkamp-Kaspers S, Hawke KL, van Driel ML. Oral medications to treat toenail fungal infection. JAMA. 2018;319:397-8. https:// doi.org/10.1001/jama.2017.20160. Good review of therapies.

30. Snell M, Klebert M, Önen N, Hubert S. A novel treatment for onychomycosis in people living with HIV infection: Vicks VapoRub $^{\mathrm{TM}}$ is effective and safe. J Assoc Nurses AIDS Care. 2016;27:109-13. https://doi.org/10.1016/j.jana.2015.10.004.

31. Rizi K, Mohammed IK, Xu K, Kinloch AJ, Charalambides MN, Murdan S. A systemic approach to the formulation of antionychomycotic nail patches. Eur J Pharm Biopharm. 2018;127: 355-65. https://doi.org/10.1016/j.ejpb.2018.02.032. Interesting proposal for new therapy development.

32. Veiga FF, Gadelha MC, da Silva MRT, Costa MI, Kischkel B, de Castro-Hoshino LV, et al. Propolis exrtract for onychomycosis topical treatment: from bench to clinic. Front Microbiol. 2018;9:779. https://doi.org/10.3389/fmicb.2018.00779.

33. Park KY, Suh JH, Kim BJ, Kim MN, Hong CK. Randomized clinical trial to evaluate the efficacy and safety of combination therapy with short pulsed 1,064 nm neodinium-doped yttrium aluminium garnet laser and amorolfine nail lacquer for onychomycosis. Ann Dermatol. 2017;29:699-705. https://doi.org/10.5021/ad.2017.29.6. 699.

34. Sonthalia S, Jakhar D, Yadav P, Kaur I. Chemical peeling as an innovative treatment alternative to oral antifungals for onychomycosis in special circumstances. Skin Appendage Disord. 2019;5:181-5. https://doi.org/10.1159/000495152. Interesting proposal for new therapy modalities.

Publisher's Note Springer Nature remains neutral with regard to jurisdictional claims in published maps and institutional affiliations. 